\title{
Effect of a filamentary discharge on the particle trajectory in a plane-to-plane DBD precipitator
}

\author{
N Zouzou and E Moreau \\ Institut Pprime, CNRS - Université de Poitiers - ENSMA, \\ SP2MI - Téléport 2 Boulevard Marie et Pierre Curie, BP 30179, \\ F86962 Futuroscope Chasseneuil Cedex, France. \\ E-mail: noureddine.zouzou@univ-poitiers.fr
}

\begin{abstract}
.
In this paper, particle velocity fields inside a plane-to-plane Dielectric Barrier Discharge (DBD) precipitator is investigated using time-resolved Particle Image Velocimetry (PIV). The main objective is to analyze the effect of a filamentary discharge on the particle trajectory. A sine wave high voltage $(24 \mathrm{kV}, 30 \mathrm{~Hz})$ is applied to create a DBD inside a planar gap $(6.4 \mathrm{~mm})$ filled with particles with a mean size of about $0.28 \mu \mathrm{m}$. The time-averaged velocity of the flow in the center of the channel is about $1 \mathrm{~m} / \mathrm{s}$.

After the establishment of the discharge several filaments cross the gap, which induce a strong effect on the particle trajectory. During a complete period of the voltage, successive phenomena are observed. Before the first filament propagation, the shape of the velocity profiles is typical of a laminar flow. At the early stage following the filament propagation across the gap, the grey-level images show a sudden disappearance of the particles at the same location where the filament takes place. This is due to the fast precipitation of particles. During the positive half-cycle, the particles migrate mainly toward the grounded electrode due to their positive net charge. At the end of a half-cycle, the polarity of the electric field is reversed then the particles initially charged return toward the channel center. Consequently, the particles oscillate delaying their collection.
\end{abstract}

\section{Introduction}

The electrostatic precipitator (ESP) is one of the most efficient particle control devices for a stationary combustion sources and a variety of industrial processes [1-5]. The ESP has the capability of capturing submicron particles at high efficiency with relatively low energy consumption and small pressure drop through the gas cleaning system.

The essential components of the ESP consist of one or more discharge electrodes of relatively small diameter (such as wires) as well as collecting electrodes (such as plates or tubes). In general, the discharge electrodes of a single stage ESP are connected to a dc high voltage, whereas the collecting electrodes are grounded.

In previous investigations, Dielectric Barrier Discharge (DBD) has been successfully used to precipitate submicron particles within the range of 0.2 to $1 \mu \mathrm{m}$ [6-7]. It has been shown that ac high voltage power supply operating at industrial frequencies (around $50 \mathrm{~Hz}$ ) is sufficient to perform the electrostatic precipitation, which can reduce the high capital costs. Beside this advantage, the DBD avoids arc transition phenomenon which can interfere with power supplies in the case of dc corona discharges.

Recently, standard low-frequency Particle Image Velocimetry (PIV) has been used in the case of plane-to-plane DBD to show that the effect of time-averaged ionic wind on the primary flow in such system was negligible [8-9]. However, the time-averaged flow was modified in the boundary layer, which became thinner. The conclusion from this set of experiments was that standard PIV was not 
adequate to make deep analysis of the electrohydrodynamic (EHD) phenomena induced by a DBD in plane-to-plane configuration.

In this paper, particle velocity fields are investigated using a time-resolved PIV system. The main objective of this study is to characterize the effect of the filamentary discharge on the particle trajectory during the positive and the negative half-cycles. Furthermore, the dynamic of the particle flow is analyzed.

In the first part of this paper, the experimental setup is described. Then, results concerning the discharge characteristics and particle velocity fields are discussed. Finally, conclusions are summarized.

\section{Experimental setup}

\subsection{ESP design}

As shown in figure 1, the plane-to-plane ESP based on a DBD consists of two parallel electrodes (aluminum foils, $80 \mathrm{~mm}$-length and $60 \mathrm{~mm}$-width in x-direction and $\mathrm{z}$-direction, respectively). The upper electrode is connected to the high voltage and the other lower is grounded. Both electrodes are wrapped on $2 \mathrm{~mm}$-thick dielectric plates (Pyrex, $600 \mathrm{~mm}$-length and $60 \mathrm{~mm}$-width in $\mathrm{x}$ and $\mathrm{z}$ directions, respectively), which form the wind tunnel used for the PIV measurements. The air gap formed by the dielectric plates is $\mathrm{L}=6.4 \mathrm{~mm}$.

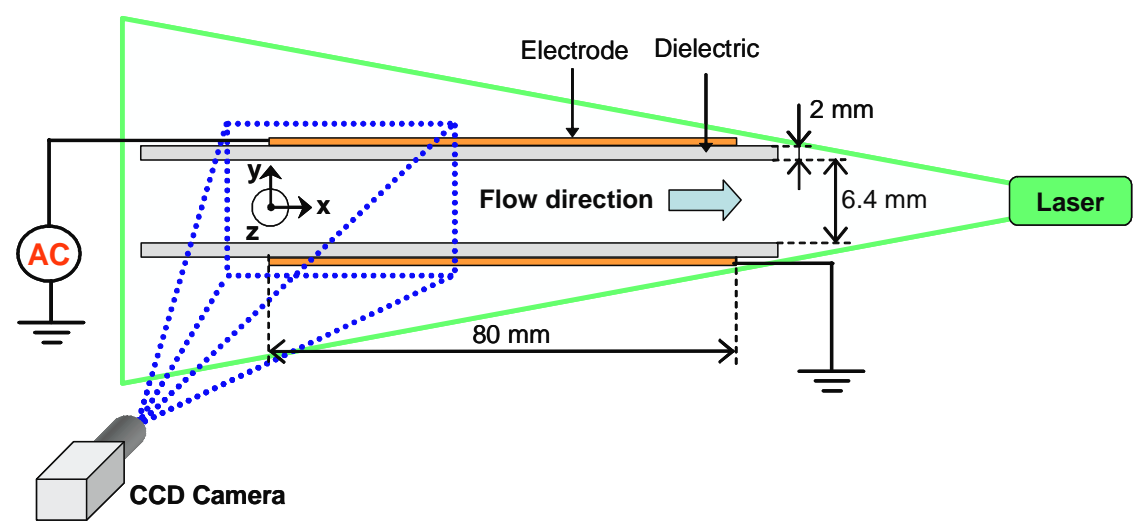

Figure 1. Schematic illustration of the experimental setup.

\subsection{Electrical devices}

The power supply system consists of a high voltage power amplifier (Trek, $30 / 20 \mathrm{C}, \pm 30 \mathrm{kV}, \pm 20 \mathrm{~mA}$ ), a signal generator (Hameg, HM 8130), a current probe (shunt resistor of $100 \Omega$ ), a high voltage probe (internal probe of the amplifier) and a digital oscilloscope (Lecroy 424, $200 \mathrm{MHz}, 2 \mathrm{GS} / \mathrm{s}$ ). The ac sine wave high voltage is applied to create the DBD inside the ESP. Several electrical conditions have been tested: two applied voltage magnitudes (21 and $24 \mathrm{kV})$ and three frequencies $(10,30$ and $100 \mathrm{~Hz})$. In this work, we present only a typical example of the time-resolved PIV results $(24 \mathrm{kV}, 30 \mathrm{~Hz})$.

A second signal generator is used as a synchronizer for triggering the time-resolved PIV system with the electrical signal supplied to the ESP.

\subsection{Particle tracking using time-resolved PIV}

Submicron particles are generated from incense burning and introduced into the precipitator in order to examine the flow modifications induced by the discharges. For that, room air is pushed by a controlled fan through a box $\left(3.5 \times 10^{-2} \mathrm{~m}^{3}\right)$ where incense sticks are burnt. Then, the formed air-incense smoke mixture flows in the wind tunnel. The average velocity at the center of the precipitator is $U_{\mathrm{o}}=1 \mathrm{~m} / \mathrm{s}$ (the Reynolds number based on the air gap length $\mathrm{L}$ is $\mathrm{Re} \approx 400$ ). 
Inside the ESP, particle velocity fields are analyzed experimentally using a time-resolved PIV system manufactured by LaVision. This system uses a dual-head high-speed laser system (Pegasus, New wave research) coupled to a 10 bit high-speed camera (Photron, APX-RS, resolution of $1376 \times 1040$ pixels). The acquisition rate is set to $6 \mathrm{kHz}$ allowing a good analysis of the particle flow dynamics. Sequences of 2000 images are recorded. With applied voltage frequency of $30 \mathrm{~Hz}$, the total acquisition time of the time-resolved PIV is about 333 ms. The velocity components are computed using a sequential cross-correlation algorithm with adaptative multipass, interrogation windows of $64 \times 64$ to $16 \times 16$ pixels and an overlap set to $50 \%$. More details about the time-resolved PIV technique can be found in [10-11].

All the experiments are carried out at atmospheric pressure and room temperature.

\section{Results and discussion}

In the following sections, typical current waveform, electrostatic precipitation and some representative particle velocity fields are discussed.

\subsection{Electrical characteristics}

Figure 2 shows the typical time evolutions of both the applied voltage and the discharge current. The discharge in the plane-to-plane DBD precipitator operates in a filamentary mode with numerous current pulses of magnitude up to $600 \mathrm{~mA}$. The current pulses, which can be easily recorded by using a shunt resistor, correspond to one or several simultaneous microdischarges crossing the gap.

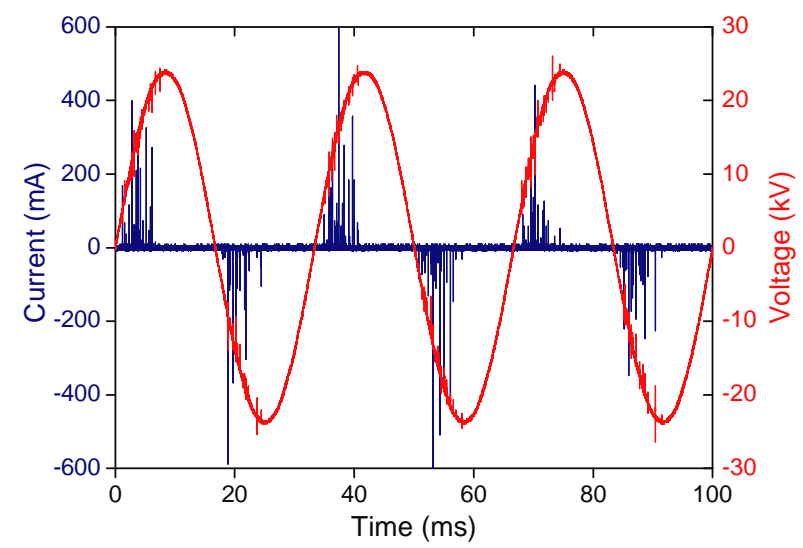

Figure 2. Time evolutions of both the applied voltage and discharge current.

Conditions: $\mathrm{V}_{\text {peak }}=24 \mathrm{kV}, \mathrm{f}=30 \mathrm{~Hz}, \mathrm{U}_{\mathrm{o}}=1 \mathrm{~m} / \mathrm{s}$.

\subsection{Electrostatic precipitation}

Figure 3 shows an example of incense particle size distribution in the outlet gas in absence of the discharge, measured using an aerosol spectrometer (Pallas Aerosoltechnologie, Model Wellas-1000). The median particle diameter is about $0.28 \mu \mathrm{m}$. The cumulative particle concentration, calculated as the sum of the values measured for each size interval between 0.1 and $1 \mu \mathrm{m}$, is about $8.2 \times 10^{5}$ particles $/ \mathrm{cm}^{3}$. The concentration level is high in order to obtain PIV images that are sufficiently luminous and weakly noised to be easily post-treated. Obviously, the collection efficiency is better when the particle concentration decreases [9]. 


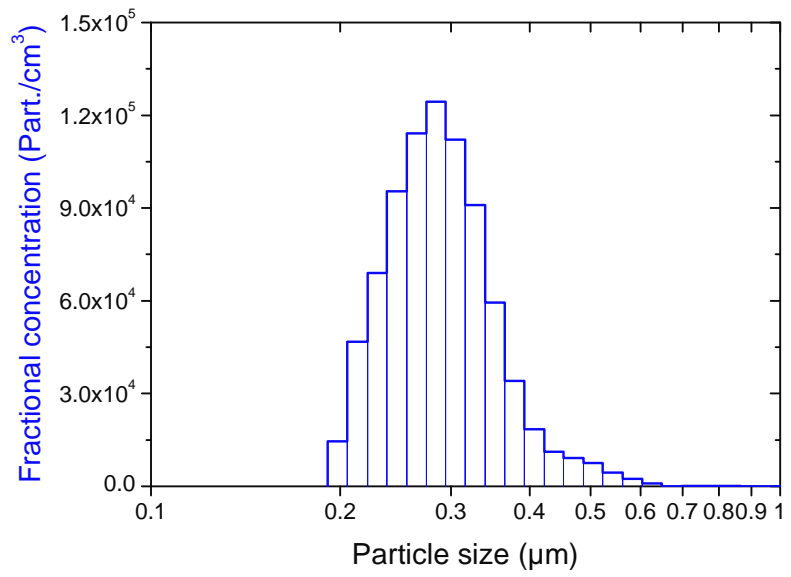

Figure 3. Typical particle size distribution of incense smoke.

\subsection{Particle Velocity fields}

Even if the acquisition duration of the time-resolved PIV is long (333 ms), only a short sequence of about $50 \mathrm{~ms}$ is presented and analyzed in this paper. Figure 4 shows few representative times of this sequence, which are corresponding to the first three half-cycles.

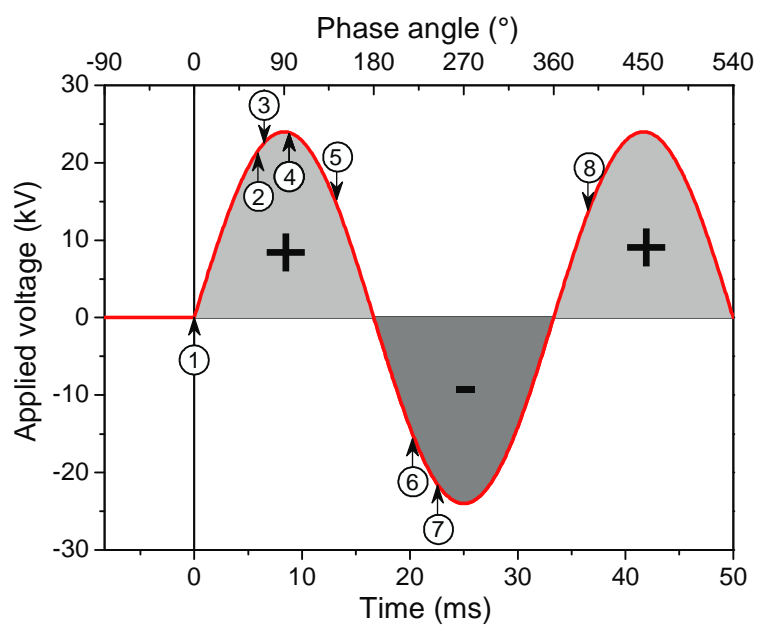

Figure 4. Representative times (phase angle) of the analyzed sequence.

The PIV images and velocity vector fields, measured using time-resolved PIV, are shown in figure 5. In order to obtain a clear description of the phenomenon, we focus on the initial part of the active area located between $\mathrm{x}=0$ and $20 \mathrm{~mm}$.

(1) $\mathrm{t}=0 \mathrm{~ms}, \theta=0^{\circ}$ (figure 5(a) and figure 5(i))

The high voltage is applied without any visible modification in the grey levels of the PIV images. The flow velocity profiles (along $\mathrm{y}$-axis) are similar all along the $\mathrm{x}$-axis. Their shape is typical of a laminar flow with $\mathrm{U}_{\mathrm{o}}=1 \mathrm{~m} / \mathrm{s}$.

(2) $\mathrm{t}=5.83 \mathrm{~ms}, \theta=63^{\circ}$ (figure $5(\mathrm{~b})$ and figure $5(\mathrm{j})$ )

The applied voltage reaches $21.4 \mathrm{kV}$ before the first filament signature. These results are similar to that observed at $\mathrm{t}=0 \mathrm{~ms}$. 

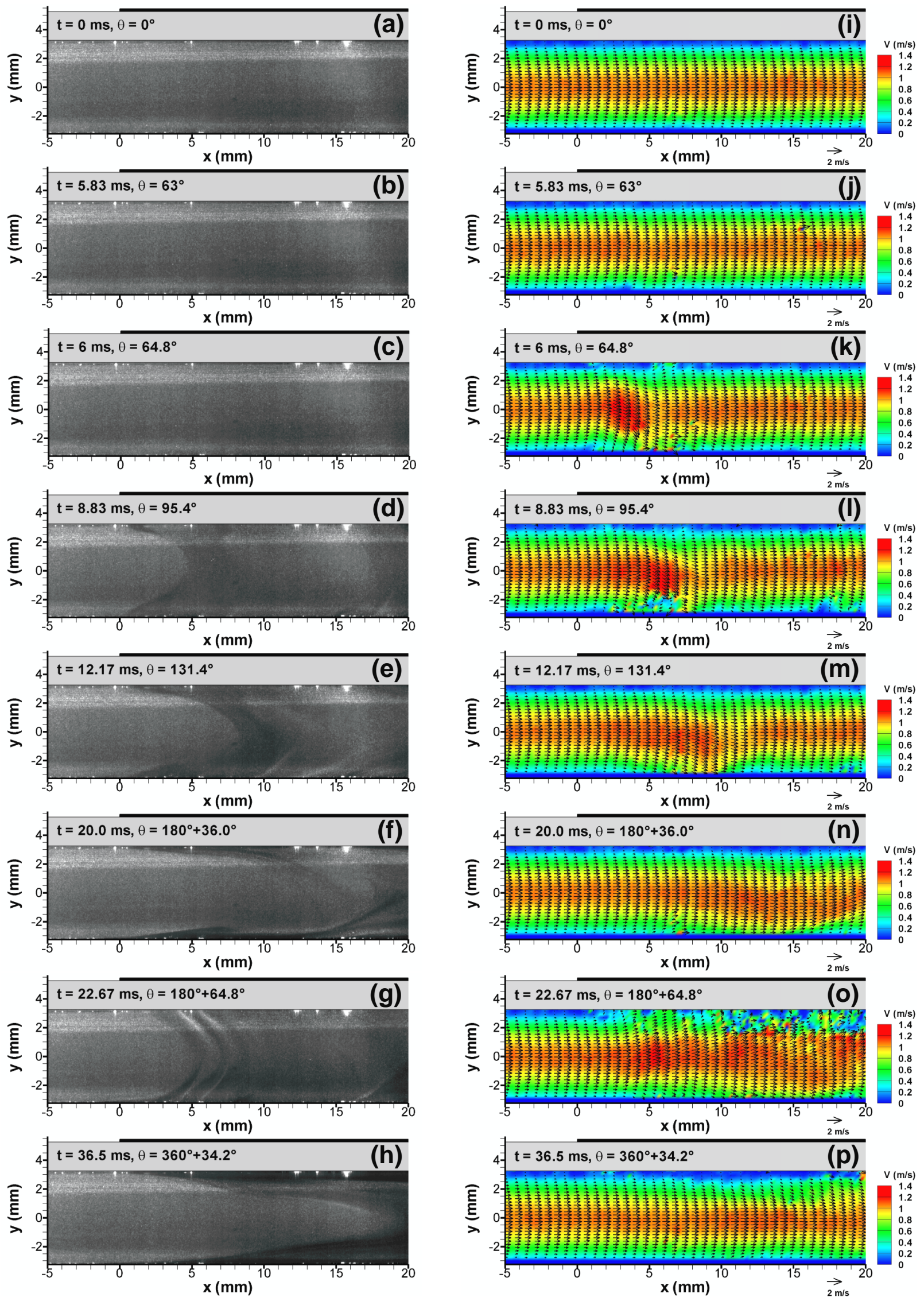

Figure 5. Grey-level images and velocity vector fields measured using time-resolved PIV. Conditions: $\mathrm{V}_{\text {peak }}=24 \mathrm{kV}, \mathrm{f}=30 \mathrm{~Hz}$, sine waveform, $\mathrm{U}_{\mathrm{o}}=1 \mathrm{~m} / \mathrm{s}$, acquisition rate $=6 \mathrm{kHz}$. 
(3) $\mathrm{t}=6 \mathrm{~ms}, \theta=64.8^{\circ}$ (figure $5(\mathrm{c})$ and figure $5(\mathrm{k})$ )

At the early stage after the filament propagation from the positive electrode towards the grounded one, the flow velocity fields exhibits a significant changes from that observed without discharge $(\mathrm{t} \leq 5.83$ $\mathrm{ms})$. Near the electrode edges $(2 \mathrm{~mm} \leq \mathrm{x} \leq 6 \mathrm{~mm})$, the strong electric forces move the charged particles from the central part of the channel toward the grounded electrode (lower one). At this moment, the upper electrode is connected to a positive high voltage. One can conclude that the particles are predominantly positively charged.

(4) $\mathrm{t}=8.83 \mathrm{~ms}, \theta=95.4^{\circ}$ (figure 5(d) and figure 5(1))

The particle concentration has strongly decreased at the location of the first filament because of the electrostatic precipitation of particles. The area where the particles have been removed flows, showing the velocity profile inside the channel (parabolic shape). Within the drift region, the particle velocity depends on the balance between the electric and the viscous forces. At this moment, the location of the charged particles is between $\mathrm{x}=4$ and $8 \mathrm{~mm}$.

(5) $\mathrm{t}=12.17 \mathrm{~ms}, \theta=131.4^{\circ}$ (figure $5(\mathrm{e})$ and figure $5(\mathrm{~m})$ )

At this stage, a great amount of charged particles have been collected. Consequently, the area at which the particle concentration is low has been expanded. In addition, the y-component of the particle velocity is reduced.

(6) $\mathrm{t}=20 \mathrm{~ms}, \theta=180^{\circ}+36^{\circ}$ (figure $5(\mathrm{f})$ and figure $5(\mathrm{n})$ )

The location of the low concentration area is now located between $\mathrm{x}=15$ and $20 \mathrm{~mm}$ because the particles are dragged by the free flow. The positively charged particle trajectories situated in this area are now bent toward the upper electrode because the electric field is reversed.

(7) $\mathrm{t}=22.67 \mathrm{~ms}, \theta=180^{\circ}+64.8^{\circ}$ (figure $5(\mathrm{~g})$ and figure $5(\mathrm{o})$ )

When the negative discharge takes place, several filaments cross the gap affecting the PIV images and the velocity fields. At least, the signature of three of them is observed between $\mathrm{x}=2$ and $8 \mathrm{~mm}$. The grey-level images show a sudden disappearance of particles at the same location where the filaments take place. Elsewhere, the high particle concentration volume seems to be expanded, inducing a rapid change of particle concentration near the boundary layers. The strength of these phenomena affects the convergence of the sequential computation of the particle velocity, especially near the upper electrode. The velocity fields show that the particles drift principally toward the upper electrode because of positive net charge. However, it seems that some amount of them flows in the opposite direction near the lower electrode between $\mathrm{x}=5$ and $20 \mathrm{~mm}$. This result will be discussed in the next section.

(8) $\mathrm{t}=36.5 \mathrm{~ms}, \theta=360^{\circ}+34.2^{\circ}$ (figure $5(\mathrm{~h})$ and figure $5(\mathrm{p})$ )

The memory effect is visible during the third half-cycle (positive one) before the appearance of new filaments. In fact, the particle concentration increases in the centre of the channel and decreases inside the boundary layers.

The polarity of the voltage really applied to the gas changes, but the main sign of the particle charge remains unchanged. This phenomenon induces observable particle oscillations when increasing the frequency (result not shown in this paper).

\subsection{Discussion}

In a typical electrostatic precipitator, particles are charged in the inter-electrode gap by the ions produced near the discharge electrodes. The electrically charged particles are then driven by the Coulomb forces due to the electric field present in the electrode gap. Their migration towards the collecting electrodes is also affected by the viscous forces associated with the fluid flow and the ionic wind [9]. Usually, the particle trajectory is three dimensional.

The particle charging and drift processes are completely different in the case of the plane-to-plane DBD ESP under study. In this case, the discharge operates in a filamentary mode. Then, several 
phenomena can be observed related to the propagation of the filaments across the gap and their interaction with submicron particles.

During the filament propagation, the particles suddenly disappear at the same location of the filament of both positive and negative half-cycles. This can be explained by a fast diffusion charging mechanism based on electron charging; hence charged particles may be collected at the wall during initial electron-positive ion plasma stage [12].

In addition, the discharge induces a fast increase of the particle concentration near the boundary layers. Then, the area of high particle concentration expands rapidly. Several reasons could be at the origin of this quick and strong phenomenon. One of them is probably related to a shockwave propagation occurring around filaments, which is in good agreement with recent investigations [13].

After the filament propagation across the gap the particles moves generally in the direction of the electric field, which means that the main charge of the particles is positive. For instance, the particle velocity fields are deflected toward the grounded electrode when a positive voltage is applied (figure 4(k), figure 4(l) and figure 4(m)). This result is mainly due to the positive ions remaining in the gap as it has been reported in the past $[14,15]$.

However, some amount of particles can migrate in the opposite direction during the early stage of the filament propagation and far from its location (figure 4(o)). These particles are not necessary negatively charged, because the pressure wave propagation could also affect the uncharged particles.

\section{Conclusion}

In this paper, particle velocity fields inside a plane-to-plane DBD precipitator have been investigated using a time-resolved PIV system. The main objective was to examine the effect of the filamentary discharge on the particle trajectory during a complete period of a sine wave high voltage.

After the discharge ignition, successive phenomena have been observed. Before the first filament propagation, the shape of the velocity profiles is the one of a typical laminar flow. At the early stage following the first filament propagation across the gap, the grey-level images show a sudden disappearance of the particles at the same location where the filament takes place, which is probably due to the contribution of electron charging mechanism during the initial electron-positive ion plasma stage. The shockwave propagation around filaments is also expected to play an important role in the observed results.

During the positive half-cycle, the particles migrate mainly toward the grounded electrode due to their positive net charge. At the end of a half-cycle, the polarity of the electric field changes then the particles initially charged return towards the channel center. Consequently, the particles oscillate, delaying their collection.

Obviously, more investigations should be conducted in order to have a deep understanding of particle charging and drift processes under a plate-to-plate dielectric barrier discharge.

\section{Acknowledgments}

The authors would like to thank J. S. Chang, A. Mizuno and G. Touchard for their helpful discussions and comments.

\section{References}

[1] Chang J S, Kelly AJ and Crowley J M 1995 Handbook of Electrostatic Processes (New York: Marcel Dekker Edition) pp 441-80

[2] Parker K R 1997 Applied Electrostatic Precipitation (London, Edition Kluwer Academic Publishers) pp 1-24.

[3] Mizuno A 2000 Electrostatic precipitation IEEE Trans. Dielectr. Electr. Insul. 7 615-24

[4] Chang J S 2003 Next generation integrated electrostatic gas cleaning systems J. Electrostat. 57 273-91

[5] Jaworek A, Krupa A, and Czech T 2007 Modern electrostatic devices and methods for exhaust gas cleaning: A brief review J. Electrostat. 65 133-55 
[6] Dramane B, Zouzou N, Moreau E. and Touchard G 2009 Electrostatic precipitation in wire-tocylinder configuration: Effect of the high-voltage power supply waveform J. Electrostat. 67117 22

[7] Dramane B, Zouzou N, Moreau E and Touchard G 2009 Electrostatic Precipitation of Submicron Particles using a DBD in axisymmetric and planar configurations IEEE Trans. Dielectr. Electr. Insul. $16343-51$

[8] Zouzou N, Dramane B, Braud P, Moreau E and Touchard G 2009 EHD Flow in DBD Precipitator International Journal of Plasma Environmental Science \& Technology 3 142-5

[9] Zouzou N, Dramane B, Moreau E and Touchard G 2011 EHD Flow and Collection Efficiency of a DBD-ESP in Wire-to-Plane and Plane-to-Plane Configurations IEEE Trans. Ind. Appl. 47336 43.

[10] Benard N, Braud P, Jolibois J and Moreau E 2008 Airflow reattachment along a NACA 0015 airfoil by surface dielectric barrier discharge actuator-time resolved particle image velocimetry investigation AIAA Paper 2008-4202

[11] Benard N and Moreau E 2011 On the Vortex Dynamic of Airflow Reattachment Forced by a Single Non-thermal Plasma Discharge Actuator Flow, Turbulence and Combustion DOI: 10.1007/s10494-011-9325-4 (in press)

[12] Chang J S, Zouzou N, Dramane B, Moreau E and Touchard G 2010 Theory of Sub-micron and Ultra-fine Dust Particle Bipolar Chargings under Dielectric Barrier Discharge Conditions Proc. SFE'2010 (Montpellier, France, 30 August - 1 September 2010)

[13] Odic E, Dhainaut M, Goldman A, Goldman M and Dessante Ph 2005 Study in space and time of the gas temperature variations in dielectric barrier discharge reactors $J$. Adv. Oxid. Technol. 8 133-41

[14] Sano Y, Kawada Y, Takahashi T, Ehara Y, Ito T, Zukeran A and Takamatsu T 2000 Diesel exhaust particles charged by barrier discharge J. Aerosol. Sci. 31 879-80

[15] Jidenko N and Borra J P 2005 Kinematics of charged nanometric particles in silent discharges $J$. Phys. D: Appl. Phys. 38 617-20 


\section{Figure Captions}

Figure 1. Schematic illustration of the experimental setup.

Figure 2. Time evolutions of both the applied voltage and discharge current. Conditions: $\mathrm{V}_{\text {peak }}=24$ $\mathrm{kV}, \mathrm{f}=30 \mathrm{~Hz}, \mathrm{U}_{\mathrm{o}}=1 \mathrm{~m} / \mathrm{s}$.

Figure 3. Typical particle size distribution of incense smoke.

Figure 4. Representative times (phase angle) of the analyzed sequence.

Figure 5. Grey-level images and velocity vector fields measured using time-resolved PIV. Conditions: $\mathrm{V}_{\text {peak }}=24 \mathrm{kV}, \mathrm{f}=30 \mathrm{~Hz}$, sine waveform, $\mathrm{U}_{\mathrm{o}}=1 \mathrm{~m} / \mathrm{s}$, acquisition rate $=6 \mathrm{kHz}$. 